UVX 2010 (2011) 245-248

DOI: $10.1051 / \mathrm{uvx} / 2011037$

(C) Owned by the authors, published by EDP Sciences, 2011

\title{
Systèmes multicouches à base d'aluminum réalisés par pulvérisation ionique dans le domaine Extrême Ultra-Violet pour l'imagerie solaire
}

\author{
A. Ziani ${ }^{1,3}$, A. Jérome ${ }^{1}$, M. Roulliay ${ }^{2}$, E. Meltchakov ${ }^{1}$, F. Bridou ${ }^{1}$, K. Gasc ${ }^{3}$ \\ et F. Delmotte ${ }^{1}$ \\ ${ }^{1}$ Laboratoire Charles Fabry de l'Institut d'Optique, Campus Polytechnique, 91127 Palaiseau, \\ France \\ ${ }^{2}$ Laboratoire d'Interaction du Rayonnement $X$ avec la Matière, Université Paris-Sud, \\ 91405 Orsay, France \\ ${ }^{3}$ Centre National d'Études Spatiales, 18 avenue E. Belin, 31401 Toulouse, France
}

\begin{abstract}
Résumé. Ce travail porte sur la conception, la réalisation et la caractérisation de nouveaux miroirs interférentiels extrême ultra-violet (EUV) pour des applications en imagerie spatiale. Ce sujet se situe dans la continuité des études menées au laboratoire Charles Fabry de L'institut d'Optique depuis de nombreuses années. Les résultats visés seront particulièrement importants pour la préparation de la mission Solar Orbiter.
\end{abstract}

\section{INTRODUCTION}

Dans le domaine de l'imagerie solaire, les systèmes multicouches usuels sont à base de silicium. Le système silicium/molybdène $[\mathrm{Si} / \mathrm{Mo}]$ étant le plus utilisé jusqu'à présent. Pour ce travail de recherche, nous avons choisi de nous orienter vers le développement de miroirs multicouches à base d'aluminium dans la bande spectrale [ $[17 \mathrm{~nm}-34 \mathrm{~nm}$ ]. Le remplacement du silicium par de l'aluminium est motivé par la faible absorption de l'aluminium dans cette zone spectrale qui permet d'obtenir une meilleure réflectivité théorique. Ceci comporte, bien entendu, de grandes difficultés technologiques, qui sont dues à la tendance de l'aluminium à cristalliser facilement, ce qui provoque de fortes rugosités aux interfaces. De plus, la forte réactivité de l'aluminium avec l'oxygène pose des problèmes d'oxydation. Les derniers miroirs réalisés au laboratoire par pulvérisation cathodique magnétron ont montré le grand intérêt des structures à base d'aluminium où les réflectivités peuvent atteindre $49.2 \%$ et $45.7 \%$ pour les longueurs d'onde $17 \mathrm{~nm}$ et $19 \mathrm{~nm}$ respectivement [1]. Le but est d'optimiser les conditions de dépôt des multicouches à base d'aluminium par pulvérisation ionique IBS (Ions Beam Sputtering). La basse pression lors du dépôt par IBS génère moins de défauts dans les multicouches par rapport à la pulvérisation cathodique [2]. De plus, la température du dépôt par faisceau d'ions est moins importe que par pulvérisation cathodique. Ceci laisse espérer des meilleures performances des multicouches. Historiquement, c'est l'IBS qui a servi pour la réalisation des miroirs embarqués sur les précédentes missions spatiales (EIT/SOHO, EUVI/STEREO, SWAP/PROBA2, HECOR/ HERSCHEL) [3].

Ce travail de recherche se place dans le contexte de la préparation de la mission spatiale Solar Orbiter. Il constitue la suite des études déjà réalisées sur des miroirs synthétisés par pulvérisation ionique (IBS) de bi-matériaux aluminium/molybdène $[\mathrm{Al} / \mathrm{Mo}$ et tri-matériaux avec ajout de carbure de bore $\left[\mathrm{Al} / \mathrm{Mo} / \mathrm{B}_{4} \mathrm{C}\right]$ [4]. Les réflectivités sur ses multicouches n'atteignent que $6 \%$ à $28 \mathrm{~nm}$ et au mieux $7.5 \%$ pour les tri-matériaux. La rugosité aux interfaces est de l'ordre de 3 à $5 \mathrm{~nm}$. De telles valeurs sont préjudiciables à l'obtention de bonnes performances des miroirs. C'est dans l'objectif de réduire ces rugosités que nous avons poursuivi cette recherche.

This is an Open Access article distributed under the terms of the Creative Commons Attribution-Noncommercial License 3.0, which permits unrestricted use, distribution, and reproduction in any noncommercial medium, provided the original work is properly cited. 


\section{DÉPÔT PAR PULVÉRISATION IONIQUE DE MULTICOUCHES À BASE D'ALUMINIUM POUR LA GAMME [17 nm-34 nm]}

Les dépôts de multicouches à base d'aluminium sont réalisés avec un bâti de dépôt par pulvérisation ionique (IBS) installé en salle blanche de classe 1000 (1000 particules par pied $\left.{ }^{3}\right)$. Les caractérisations structurelles et les performances optiques des miroirs réalisés sont effectuées respectivement à l'aide d'un réflectomètre des rayons $\mathrm{X}$ en incidence rasante à $0,154 \mathrm{~nm}$ et d'un réflectomètre EUV à source plasma laser pour caractériser les valeurs de réflectivités en relatif.

Dans un premier temps, nous nous sommes intéressés à la calibration des différentes cibles ( $\mathrm{Al}, \mathrm{Mo}$ et $\mathrm{B}_{4} \mathrm{C}$ ). Pour cela, nous avons testé plusieurs configurations, en allant de l'influence de l'atmosphère de dépôt jusqu'à la variation de l'angle de bombardement par le faisceau d'ions par rapport à la cible. Nous nous sommes intéressés plus particulièrement au dépôt de l'aluminium. Les autres matériaux étant déjà maîtrisés au laboratoire [3]. Nous varions le courant du faisceau d'ions ( $\mathrm{I}_{\text {canon }}$ ) de $20 \mathrm{~mA}$ à $70 \mathrm{~mA}$. Les mesures en réflectométrie $X$ rasant montent une augmentation du nombre et de l'intensité des pics de diffraction en fonction de l'augmentation de la tension au bord du canon. Cette progression est continue et s'accompagne d'une amélioration de la rugosité du dépôt. La rugosité de surface passe de $4.55 \mathrm{~nm}$ (pour $\mathrm{I}_{\text {canon }}=20 \mathrm{~mA}$ ) à $2.3 \mathrm{~nm}$ (pour $\mathrm{I}_{\text {canon }}=70 \mathrm{~mA}$ ).

Ensuite, nous nous sommes intéressés à l'influence de la position de la cible, par rapport au canon (faisceau d'ions), sur la qualité du dépôt d'aluminium. Les valeurs des angles que nous donnons correspondent à l'écart par rapport à la position horizontale de la cible. Dans la configuration initiale, l'angle entre le canon et la cible est de $30^{\circ}$ (voir figure 1(a)). Dans cette configuration, il a été rapporté dans la littérature que l'angle d'émission des éléments pulvérisés $\phi_{0}$ variait entre $15^{\circ}$ à $30^{\circ}[5,6]$. Une étude a été réalisée au laboratoire dans le cadre de la mission STEREO sur la répartition du dépôt sur le substrat en mode statique (porte substrat non tournant) [7]. Cette étude a montré que le maximum d'épaisseur du dépôt était décalé de 7 à $9 \mathrm{~cm}$ par rapport au centre. Des calculs de l'angle d'émission nous donnent une valeur d'angle $\phi_{0}$ (Fig. 1(a)) comprise entre $14^{\circ}$ et $18^{\circ}$.

$$
\varphi_{0}=\operatorname{arrtan} x_{\max } / d
$$

Avec $x_{\text {max }}$ : la position décentrée du maximum de dépôt (dans notre cas entre $7 \mathrm{~cm}$ et $9 \mathrm{~cm}$ ) d: la distance entre la cible et le substrat $(28 \mathrm{~cm})$.

Lorsque la cible est positionnée à $15^{\circ}$, cela correspond à un angle de $45^{\circ}$ entre la cible et le canon (Fig. 1(b)). La position de la cible par rapport au canon agit sur la direction du lobe de particules pulvérisées dans le bâti. Contrôler la position de la cible permet de rediriger un maximum d'émission vers l'échantillon.

D'après les mesures de réflectométrie rayon X (Fig. 2), nous constatons que l'augmentation de l'inclinaison de la cible améliore considérablement la qualité du dépôt. On passe d'une rugosité d'interface de $3.65 \mathrm{~nm}$ pour la position $0^{\circ}$ à un peut près $1.35 \mathrm{~nm}$ pour la position $15^{\circ}$. Au-delà de
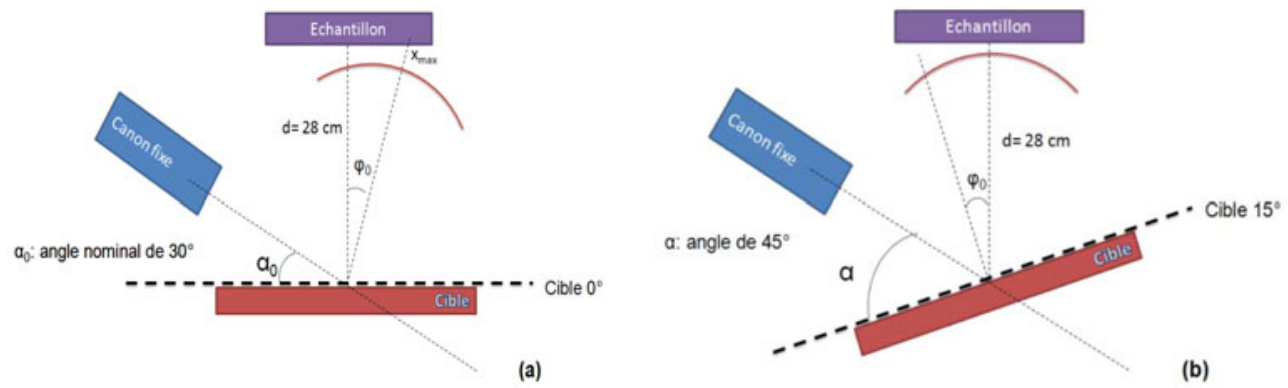

Figure 1. Schéma géométrique représentant les angles de pulvérisation de la cible. 
cette position, l'amélioration s'arrête et on risque de détériorer le canon car on se trouve dans une configuration où l'on renvoie du dépôt sur le canon.

Avec la cible inclinée à $15^{\circ}$, nous effectuons un centrage du lobe d'émission sur l'échantillon. Le dépôt se fait plus rapidement et les interfaces se trouvent améliorées. Nous avions constaté un effet similaire d'amélioration de la qualité des films minces d'aluminium avec la vitesse de dépôt en pulvérisation cathodique magnétron [1].

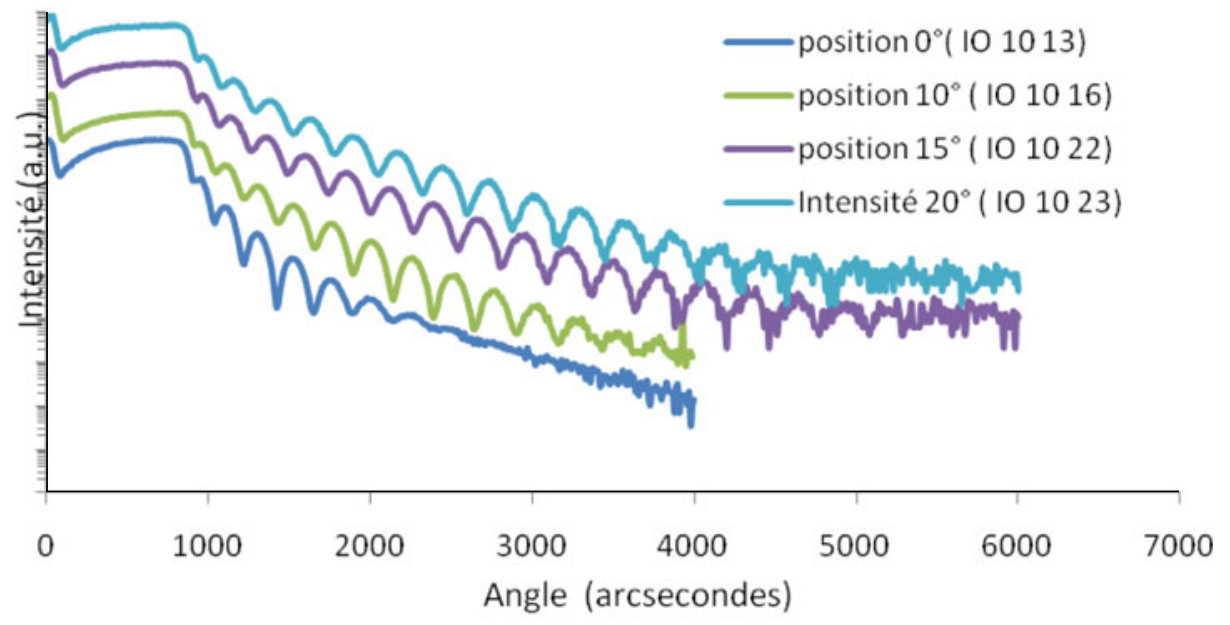

Figure 2. Mesures en réflectometrie des rayons $\mathrm{X}$ rassant d'echantillons réalisés avec différents angles de la cible d'aluminium.

Nous avons optimisé des dépôts d'aluminium fonctionnant à fort courant de canon sous atmosphère Argon/Hydrogène. La cible est inclinée de $15^{\circ}$ par rapport à l'horizontale. Ces conditions de dépôt nous permettent d'obtenir des couches d'aluminium avec une rugosité de l'ordre de $1.35 \mathrm{~nm}$ selon des simulations réalisées sur le logiciel IMD.

\section{MESURES DE RÉFLECTIVITÉ DES MULTICOUCHES [AL/MO] ET [AL/MO/B4C] DÉPOSÉES PAR IBS}

Nous avons sélectionné les meilleurs échantillons multicouches avec 15 périodes réfléchissants autour de la longueur d'onde $30 \mathrm{~nm}$. Les multicouches $[\mathrm{Al} / \mathrm{Mo}]_{15}$ présentes une rugosité de l'ordre de $1.3 \mathrm{~nm}$. Celles en tri-matériaux $\left[\mathrm{Al} / \mathrm{Mo} / \mathrm{B}_{4} \mathrm{C}\right]_{15}$ ont une rugosité de l'ordre de $2.5 \mathrm{~nm}$. Les mesures de réflectivités sont obtenues par réflectométrie EUV à source plasma laser. La précision de cette technique est limitée par le faible flux de photons de la source. Elle est généralement complétée par des mesures sur des lignes synchrotron. Dans un premier temps, et pour une première estimation des performances en réflectivité des échantillons, cette technique est suffisante.

Les valeurs de réflectivité obtenues sur les multicouches $[\mathrm{Al} / \mathrm{Mo}]_{15}$ sont très encourageantes. D'après nos études précédentes, l'ajout d'un troisième matériau dans la structure devrait permettre d'atteindre des réflectivités plus élevées. La valeur de réflectivité simulée pour une multicouches $[\mathrm{Al} / \mathrm{Mo} / \mathrm{B} 4 \mathrm{C}]_{15}$ pour la longueur d'onde réfléchie de $30.3 \mathrm{~nm}$, et sans tenir compte de la rugosité, est autour de $45 \%$. Cependant, la mesure faite sur la multicouche $\left[\mathrm{Al} / \mathrm{Mo} / \mathrm{B}_{4} \mathrm{C}\right]_{15}$ pour la longueur d'onde $30.3 \mathrm{~nm}$ n'est que de $18.2 \%$. L'ajout de $\mathrm{B}_{4} \mathrm{C}$ a eu pour effet l'augmentation de la rugosité de $1.3 \mathrm{~nm}$ à $2.5 \mathrm{~nm}$. Sur la courbe de réflectivité de rayons $\mathrm{X}$, nous observons un effondrement des pics de réflectivité et une décroissance rapide du fond de la courbe. 
IO $1047\left[\mathrm{Al} / \mathrm{Mo} / \mathbf{B}_{4} \mathrm{C}\right]_{15}$

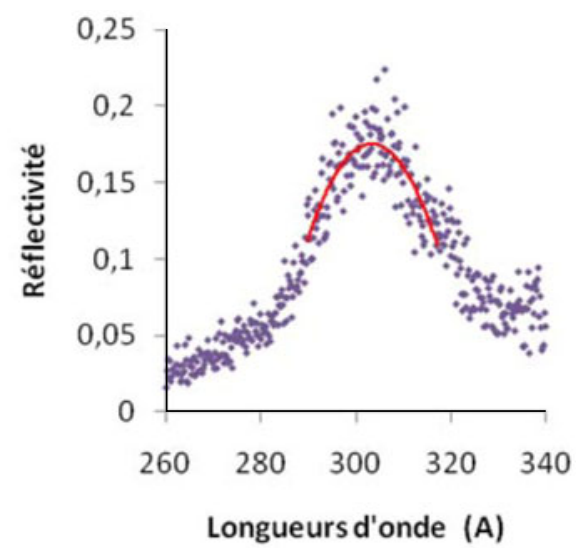

IO $1031[\mathrm{Al} / \mathrm{Mo}]_{15}$

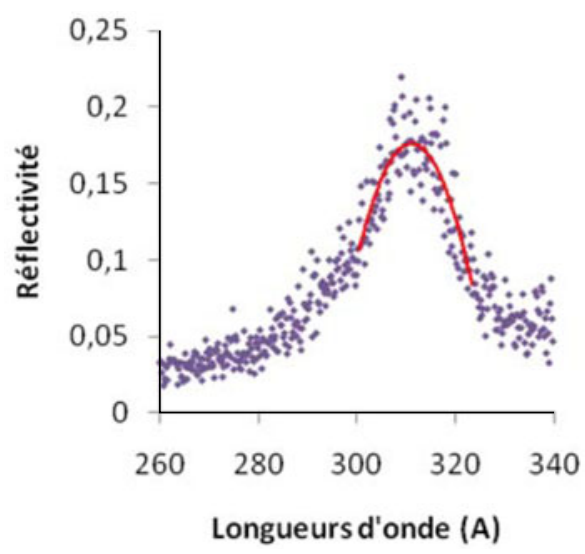

Figure 3. Réflectivités des multicouches $[\mathrm{Al} / \mathrm{Mo}$ ] et $[\mathrm{Al} / \mathrm{Mo} / \mathrm{B} 4 \mathrm{C}]$.

Les deux multicouches réfléchissent autour de $18 \%$ pour la longueur d'ondes $30.2 \mathrm{~nm}$. En quelque sorte, l'effet tri-matériau ici sert à compenser les pertes due à la rugosité, ce qui maintient la réflectivité à $18 \%$.

Selon les simulations, les multicouches $\left[\mathrm{Al} / \mathrm{Mo} / \mathrm{B}_{4} \mathrm{C}\right]$ présentent les plus forte réflectivités, mais, au contact de l'aluminium, le $\mathrm{B}_{4} \mathrm{C}$ induit une augmentation de la rugosité. Une amélioration des conditions du dépôt est envisagée. Mais dans un premier temps, nous allons remplacer le carbure de bore $\mathrm{B}_{4} \mathrm{C}$ par le carbure de silicium SiC. En effet, les multicouches $[\mathrm{Al} / \mathrm{Mo} / \mathrm{SiC}]$ présentent des performances théoriques légèrement inférieures, mais en pratique, le $\mathrm{SiC}$ se comporte mieux au contact de l'aluminium (rugosité plus faible). Nous avons entrepris aussi des séries d'analyse en microscopie électronique en transmission MET. Les images de microscopie couplées à des clichés de plans de diffraction ont montré une croissance colonnaire de l'Aluminium. Ce type de croissance est source de rugosité des multicouches et par conséquent, perte de réflectivité.

\section{CONCLUSION}

Au jour d'aujourd'hui, nous avons réussi l'optimisation du dépôt des multicouches à base d'aluminium sur IBS en fonction de plusieurs paramètres comme le courant du faisceau et de l'angle d'inclinaison de la cible. Nous avons réussi à avoir de bonnes valeurs de réflectivité sur les empilements bi-matériaux $\left[\mathrm{Al} / \mathrm{Mo}\right.$ et tri-matériau $\left[\mathrm{Al} / \mathrm{Mo} / \mathrm{B}_{4} \mathrm{C}\right]$. On obtient des réflectivités de l'ordre de $18 \%$ à $30.4 \mathrm{~nm}$. Cela reste néanmoins inférieur aux valeurs obtenues par pulvérisation cathodique (35\% dans les meilleures conditions). Nous travaillons à une meilleure optimisation des paramètres du dépôt et aussi à l'idée de traitement post-dépôt pour améliorer les réflectivités des miroirs.

\section{Références}

[1] E. Meltchakov et al. Appl. Phys. A (2010) 98: 111-117

[2] P.A. Kearney et al. J. Vac. Sci. Technol. B (1997) 15: 2452-2454

[3] M-F. Ravet et al. Proceedings of SPIE St Etienne (2003) 5250-12

[4] Compte rendu final destiné au CNES, Action R\&T: R-S07 / SU-0004-010

[5] T. Motohiro et Y. Taga Thin Solid Films (1984) 120: 313-327

[6] D. Bouchier et A. Bosseboeuf Appl. Phys. A (1991) 53: 179-184

[7] Etude réalisée par F. Bridou dans le cadre de la mission STEREO (2001, non-publié) 